\title{
Complicaciones Urológicas Tempranas del Carcinoma de Cervix Avanzado (E-III-IV) Estudio Computarizado
}

\author{
Dr. Carlos E. Vargas Cabrera*
}

\section{INTRODUCCION}

El carcinoma del cervix uterino es el cáncer que ocupa el primer puesto de los cánceres en Colombia y también en la mortalidad general del cáncer, que es a su vez la segunda causa de mortalidad en este país (1). Uno de los aspectos más importantes y menos conocidos de la supervivencia en el carcinoma de cervix es el compromiso del sistema urinario (2).

\section{Relaciones Anatómicas}

Es importante recordar las relaciones existentes entre los sistemas urológico y genital interno femenino. El uréter distal entra a la pelvis cruzando la arteria ilíaca común justamente por dentro de su bifurcación; luego desciende a lo largo de la pared pélvica lateral retroperitonealmente al lado de los vasos hipogástricos. Sigue un trayecto medial y anterior para entrar a la base del ligamento ancho. En el fórnix vaginal, aproximadamente dos $\mathrm{cms}$. lateral al cervix, el uréter está debajo de la arteria uterina y encima de la arteria vaginal. Aquí continúa a través del canal vascular de la fascia útero vaginal cruzando la vagina anterior y superior para entrar en el trigono vesical. Los linfáticos de la porción pélvica del uréter usualmente se unen a aquellos de la cara posterior de la vejiga y se vacían en los nódulos linfáticos ilíacos internos y primitivos.

Aquellos linfáticos de la cara posterior de la vejiga se anastomosan con los linfáticos del cervix y de la vagina superior y se vacían en los nódulos ilíacos internos.

* Servicio de Urología, Instituto Nacional de Cancerología.
El carcinoma de cervix invade por extensión directa el cuerpo uterino, la vagina y los parametrios y en el tracto urinario obstruye los uréteres e invade la mucosa vesical. La invasión linfática es del $47 \%$ en el estado III a los ganglios ilíacos externos, internos, primitivos, obturadores y parametriales (3).

Clasificación Internacional del

Cáncer de Cervix

$\begin{array}{ll}\text { Estado 0 = } & \text { In situ. } \\ \text { Estado I }= & \text { Confinado al cuello. } \\ \text { Estado II }= & \text { Se extiende más allá del cuello pe- } \\ & \text { ro no hasta la pared pélvica y } / 0 \\ & 2 / 3 \text { proximales de vagina. } \\ \text { Estado IIIA }= & \begin{array}{l}\text { Invasión del tercio inferior de va- } \\ \text { gina. }\end{array} \\ \text { Estado IIIB = } & \begin{array}{l}\text { Extensión hasta la pared pélvica } \\ \text { ysto hidronefrosis o riñón excluido. }\end{array} \\ \text { Estado IVA }= & \text { Invasión a mucosa vesical o rectal. } \\ \text { Estado IVB }= & \text { Invasión a órganos distales. }\end{array}$

Implicaciones del compromiso urológico

En el manejo rutinario de estas pacientes hemos observado una alta rata de complicaciones urológicas causadas por la enfermedad y por la radioterapia, que afectan el bienestar de estas pacientes impidiéndoles ser tratadas. Por otro lado en las pacientes post-radioterapia, curadas de su cáncer, quedan secuelas urológicas que conducen a la pérdida de un riñón o a la muerte y también a hematurias que pueden llevar a un estado fatal.

No tenemos un análisis estadístico de estos problemas de ocurrencia diaria que son parte del moti- 
vo de este trabajo. Se tendrán en cuenta las complicaciones urológicas pre-radioterapia en el cáncer de cervix estados III y IV y también estas mismas complicaciones en el primer año post-tratamiento. Consideraciones que son fundamentales para la clasificación del carcinoma, su tratamiento, su pronóstico, que es completamente diferente cuando hay compromiso urológico. Además del análisis interno se comparará la rata de anormalidades urográficas y cistoscópicas con series de otros países.

Hace 4 años diseñamos una urografía "simplificada", para el diagnóstico del compromiso uretrorenal en el carcinoma de cervix en todos los estados, con menos placas de las habituales para una urografía corriente, a saber:

$$
\begin{aligned}
& \text { A - Placa simple. } \\
& \text { B - Placa de } 15 \text { minutos. } \\
& \text { C - Placa vertical de } 25 \text { minutos. } \\
& \text { D - Placa post-miccional. }
\end{aligned}
$$

Se confrontará con los resultados de las urografías corrientes en otros países.

Haremos también una correlación de los hallazgos cistoscópicos con los demostrados en la urografía simplificada.

Compararemos la mortalidad de las pacientes tratadas adecuadamente que mueren por causa urológica o por otras causas. Se analizarán también las cistoscopias, urografías y síntomas de algunas pacientes con anormalidades previas al tratamiento detectadas en estos exámenes, con estos mismos estudios después de la radioterapia. Igualmente las gamagrafías renales y las biopsias vesicales de las pacientes que tuvieron indicación para estos estudios.

El análisis estadístico de los datos obtenidos fue llevado a cabo en un computador Apple IIe. Así mismo, las gráficas presentadas se elaboraron a partir de dicho análisis en el mismo computador.

\section{MATERIAL Y METODOS}

El Instituto Nacional de Cancerología atendió en el año de 19828.586 casos nuevos, de los cuales 3.480 tenían cáncer. Entre ellos 2.312 mujeres y 1.168 hombres. Se hizo el diagnóstico de carcinoma de cervix a 895 pacientes que corresponden al $25.7 \%$ de todos los pacientes con cáncer y al $38.7 \%$ de las mujeres con lo mismo.

El número de pacientes con carcinoma de cervix se discriminó por estados, así:

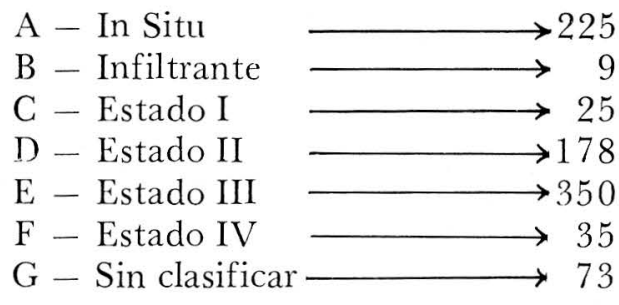

Se revisaron 367 historias clínicas con diagnóstico de carcinoma de cervix, las cuales antes de ser vistas en la consulta de Urología fueron clasificadas al menos por tres examinadores independientes, así: Consulta de admisión, Ginecología y Radioterapia. Todas las pacientes tenían diagnóstico citológico e histológico. A 236 pacientes $(82.2 \%)$ se les practicó urografía pre tratamiento y a $31(10.8 \%)$ pacientes se les practicó urografía post-tratamiento. Igualmente se les practicó cistoscopia previa al tratamiento a 266 pacientes $(92.6 \%)$ y post-tratamiento a 27 pacientes $(9.4 \%)$.

A algunas pacientes con razón de la indicación médica se les practicó gamagrafía renal y biopsia vesical. Los estudios Post-radioterapia fueron indicados en pacientes con anormalidades urológicas pre-tratamiento. Se hizo un análisis de la sintomatología urinaria baja y hematuria pre y post radioterapia y el tratamiento recibido para ello.

Todas las pacientes fueron estudiadas con cuadro hemático, parcial de orina, nitrógeno ureico, creatinina, glicemia y radiografía de tórax. Todas las pacientes motivo de este estudio recibieron radioterapia pélvica, con una dosis mínima de 8000 RADS en punto "A”.

Todas las pacientes con sintomatología urinaria baja fueron tratadas con antibióticos y analgésicos urinarios. Las pacientes con insuficiencia renal severa fueron tratadas con radioterapia y hemodiálisis concomitantemente.

Tanto para facilitar el análisis estadístico de los datos como para una mejor comprensión del estudio, las alteraciones observadas en la cistoscopia y en la urografía fueron codificadas así:

\section{Cistoscopia}

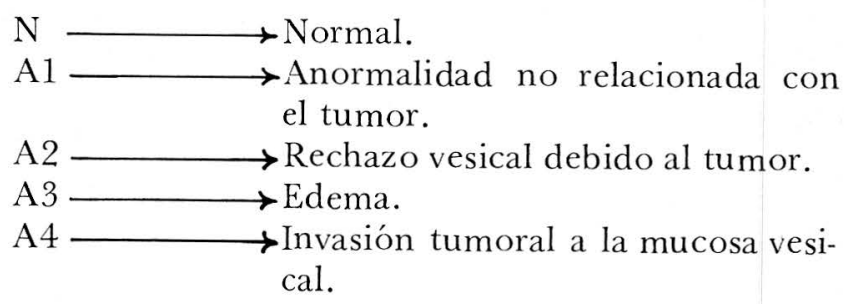


Urografía

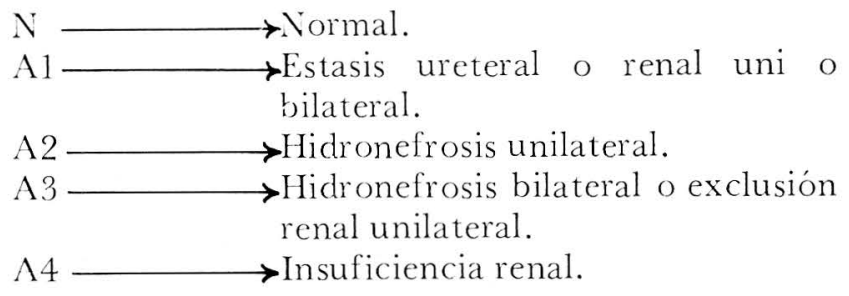

Se llevó a cabo un seguimiento de todas las pacientes desde el momento de terminar su tratamiento radioterápico hasta un año después del mismo.

Las pacientes para su estudio fueron divididas en tres grupos de edades a saber: menores de 40 años, 40-60 años y mayores de 60 años. También se tuvieron en cuenta los estados clínicos IIIA, IIIB, IVA. IVB y se cruzaron estas variables con los índices de mortalidad de causa urológica, con los resultados urográficos y cistoscópicos. Además se correlacionaron entre sí los resultados urográficos y cistoscópicos. También se estudiaron las pacientes con respecto a la sintomatología urinaria baja, hematuria y biopsias vesicales positivas.

\section{RESULTADOS}

Del total de las 367 historias revisadas se desecharon 80 (21.7), por los siguientes motivos: 50 pacientes por abandono de tratamiento (13.6\%); 25 por deficiencias estadísticas $(6.8 \%)$ y 5 pacientes por muerte antes de iniciar tratamiento (1.3\%). Por lo tanto el análisis se centró en 287 pacientes $(78.2 \%)$.

La siguiente es la agrupación de los 287 casos, discriminados por estado clínico y grupos de edad:

TABLA 1

ESTADO CLINICO - EDAD

\begin{tabular}{|lrrrrrrr|}
\hline Estado & Totales & $\begin{array}{c}<0 \text { años } \\
\text { No. }(\%)\end{array}$ & $\begin{array}{r}40-60 \text { años } \\
\text { No. }(\%)\end{array}$ & $\begin{array}{r}>0 \text { años } \\
\text { No. }(\%)\end{array}$ \\
\hline IIIA & 3 & 0 & & 1 & $(33.3)$ & 2 & $(66.6)$ \\
IIIB & 269 & 41 & $(15.2)$ & 159 & $(59.1)$ & 69 & $(25.6)$ \\
IVA & 1 & 0 & & 1 & $(100)$ & 0 & \\
IVB & 14 & 0 & & 7 & $(50)$ & 7 & $(50)$ \\
\hline Totales & 287 & 41 & $(14.2)$ & 168 & $(58.5)$ & 78 & $(27.1)$ \\
\hline
\end{tabular}

En tabla 2 mostramos la correlación que se observó entre biopsia vesical pre-radioterapia y estado clínico:

\author{
GRAFICA 1 \\ PACIENTES - EDAD
}

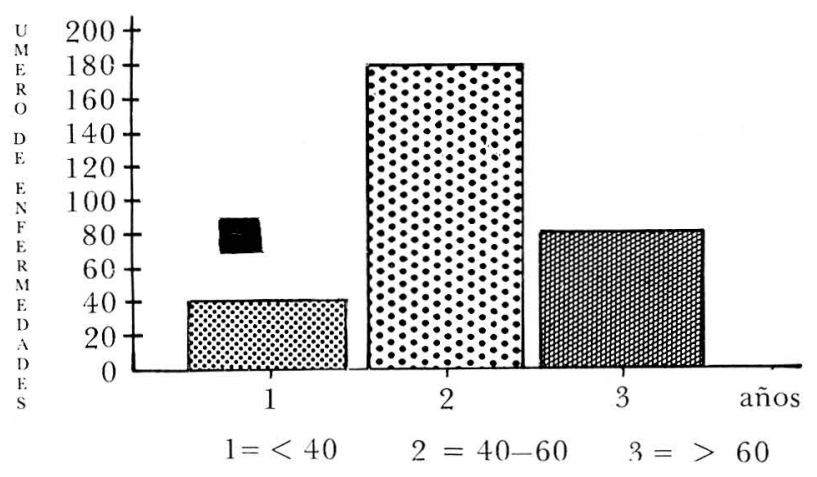

TABLA 2

BIOPSIA VESICAL PRE-RADIO Y ESTADO CLINICO

\begin{tabular}{|lcllll|}
\hline $\begin{array}{c}\text { Resultado } \\
\text { Biopsia }\end{array}$ & Totales & $\begin{array}{l}\text { IIIA } \\
\text { No. }(\%)\end{array}$ & $\begin{array}{l}\text { IIIB } \\
\text { No. }(\%)\end{array}$ & $\begin{array}{l}\text { IVA } \\
\text { No. }(\%)\end{array}$ & $\begin{array}{l}\text { IVB } \\
\text { No. }(\%)\end{array}$ \\
\hline Positivo & 28 & $1(3.5)$ & $21(75)$ & 0 & $6(21.4)$ \\
Negativo & 25 & 0 & $25(100)$ & 0 & 0 \\
\hline Totales & 53 & $1(1.8)$ & $46(86.7)$ & 0 & $6(11.3)$ \\
\hline
\end{tabular}

La siguiente tabla nos muestra el número de pacientes cuyo estado clínico varió después de la cistoscopia pre-radioterapia:

TABLA 3

\section{ESTADO CLINICO PRE Y POST CISTOSCOPIA}

\begin{tabular}{|ccc|}
\hline Estado Pre-Cistoscopia & Estado Post-Cistoscopia & Total \\
\hline III B & IV A & 21 \\
III A & IV A & 1 \\
\hline
\end{tabular}

De la comparación entre la urografía pre-radioterapia y el estado clínico obtuvimos: (Ver tabla 4 ).

GRAFICA 2

\section{UROGRAFIA / PACIENTES}

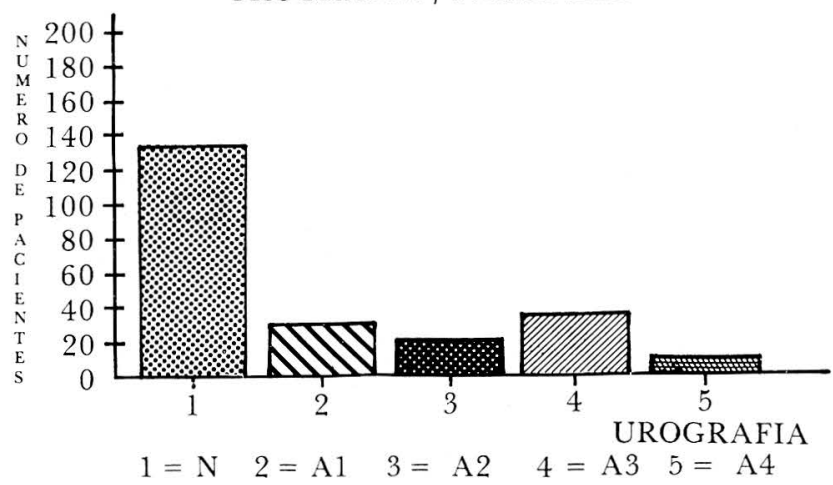


TABLA 4

LROGRAFIA PRE-RADIOTERAPIA / ESTADO CLINICO

\begin{tabular}{|lclllll|}
\hline Estado & Totales & $\begin{array}{l}\text { Uro.N. } \\
\text { No. }(\%)\end{array}$ & $\begin{array}{l}\text { Uro. A1. } \\
\text { No. }(\%)\end{array}$ & $\begin{array}{l}\text { Uro. A2. } \\
\text { No. }(\%)\end{array}$ & $\begin{array}{l}\text { Uro. A3. } \\
\text { No. }(\%)\end{array}$ & $\begin{array}{l}\text { Uro A4. } \\
\text { No. }(\%)\end{array}$ \\
\hline III A & 3 & $1(33.3)$ & $1(33.3)$ & 0 & $1(33.3)$ & 0 \\
III B & 224 & $132(58.9)$ & $29(12.9)$ & $22(9.8)$ & $32(14.2)$ & $9(4)$ \\
IVA & 0 & 0 & 0 & 0 & 0 & 0 \\
IVB & 9 & $1(11.1)$ & $1(11.1)$ & $2(22.2)$ & $3(33.3)$ & $2(22.2)$ \\
\hline Totales & 236 & $134(56.7)$ & $31(13.1)$ & $24(10.1)$ & $36(15.2)$ & $11(4.6)$ \\
\hline
\end{tabular}

TABLA 5

UROGRAFIA PRE-RADIOTERAPIA / EDAD

\begin{tabular}{|lclllll|}
\hline $\begin{array}{l}\text { Grupos } \\
\text { Edad }\end{array}$ & Totales & $\begin{array}{l}\text { Uro.N. } \\
\text { No. }(\%)\end{array}$ & $\begin{array}{l}\text { Uro. A1. } \\
\text { No. }(\%)\end{array}$ & $\begin{array}{l}\text { Uro. A2. } \\
\text { No. ( } \%)\end{array}$ & $\begin{array}{l}\text { Uro. A3. } \\
\text { No. (\%) }\end{array}$ & $\begin{array}{c}\text { Uro. A4. } \\
\text { No. }(\%)\end{array}$ \\
\hline$<40$ & 37 & $19(51.3)$ & $+(10.6)$ & $4(10.6)$ & $9(24.3)$ & $1(2.7)$ \\
$40-60$ & 133 & $79(59.3)$ & $17(12,7)$ & $12(9)$ & $16(12)$ & $9(6.7)$ \\
$>60$ & 66 & $36(54.5)$ & $10(15.1)$ & $8(12.1)$ & $11(16.6)$ & $1(1.5)$ \\
\hline Totales & 236 & $134(56.7)$ & $31(13.1)$ & $24(10.1)$ & $36(15.2)$ & $11(4.6)$ \\
\hline
\end{tabular}

Se obtuvieron los siguientes resultados al hacer el cruce entre urografía pre-radioterapia y grupos de cdad: (Ver tabla 5)

GRAFICA 3

UROGRAFIA / EDAD

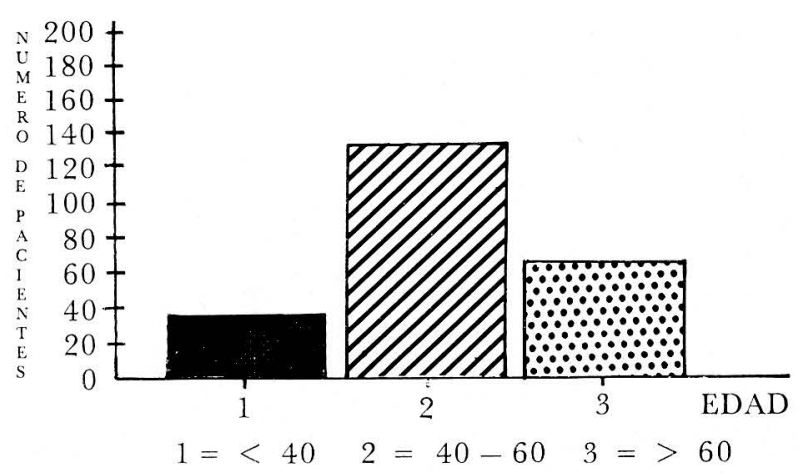

La tabla 6 muestra la agrupación de las pacientes discriminadas por estado clínico y cistoscopia preradioterapia.
GRAFICA 4

CISTOSCOPIA / PACIENTES

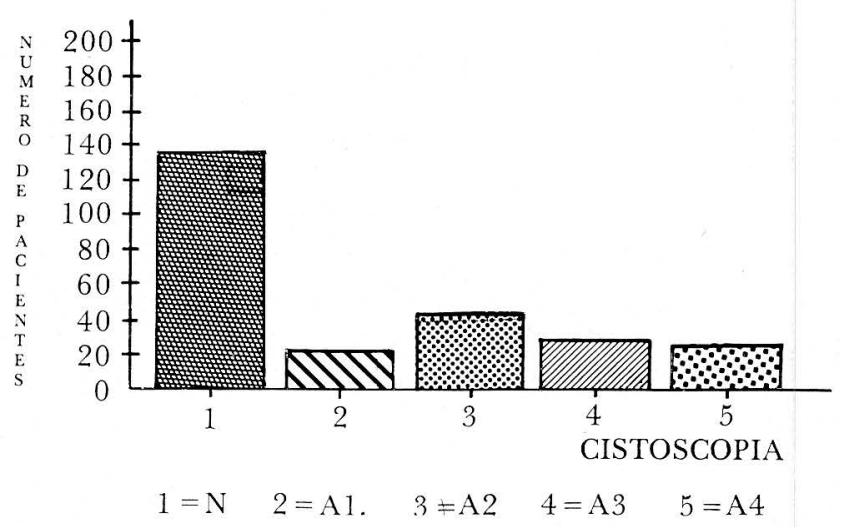

Estudiada la distribución por cistoscopia pre-radioterapia y grupos de edad, se obtuvo: (Ver tabla 7)

Realizado el cruce entre las urografías y cistoscopias pre-radioterapias encontramos: (Ver tabla 8) 
TABLA 6

ESTADO CLINICO / CISTOSCOPIA PRE-RADIOTERAPIA

\begin{tabular}{|lclllll|}
\hline Estado & Totales & $\begin{array}{l}\text { Cis. N. } \\
\text { No. }(\%)\end{array}$ & $\begin{array}{l}\text { Cis. A1. } \\
\text { No. }(\%)\end{array}$ & $\begin{array}{l}\text { Cis. A2. } \\
\text { No. }(\%)\end{array}$ & $\begin{array}{l}\text { Cis. A3. } \\
\text { No. }(\%)\end{array}$ & $\begin{array}{l}\text { Cis. A4 } \\
\text { No. }(\%)\end{array}$ \\
\hline III A & 3 & $2(66.6)$ & 0 & 0 & 0 & $1(33.3)$ \\
III B & 249 & $130(52.2)$ & $25(10)$ & $43(17.2)$ & $30(12)$ & $21(8.4)$ \\
IVA & 1 & $1(100)$ & 0 & 0 & 0 & 0 \\
IVB & 13 & $2(15.3)$ & 0 & $3(23)$ & $2(15.3)$ & $6(46.1)$ \\
\hline Totales & 266 & $135(50.7)$ & $25(9.3)$ & $46(17.2)$ & $32(12.0)$ & $28(10.5)$ \\
\hline
\end{tabular}

TABLA 7

CISTOSCOPIA PRE-RADIOTERAPIA / EDAD

\begin{tabular}{|lclllll|}
\hline $\begin{array}{c}\text { Grupos } \\
\text { Edad }\end{array}$ & Totales & $\begin{array}{l}\text { Cis. N. } \\
\text { No. }(\%)\end{array}$ & $\begin{array}{l}\text { Cis. A1. } \\
\text { No. }(\%)\end{array}$ & $\begin{array}{l}\text { Cis. A2. } \\
\text { No. }(\%)\end{array}$ & $\begin{array}{l}\text { Cis. A3. } \\
\text { No. }(\%)\end{array}$ & $\begin{array}{l}\text { Cis. A4. } \\
\text { No. }(\%)\end{array}$ \\
\hline$<40$ & 37 & $23(62.1)$ & $2(5.4)$ & $8(21.6)$ & $2(5.4)$ & $2(5.4)$ \\
$40-60$ & 154 & $77(50)$ & $11(7.1)$ & $27(17.5)$ & $24(15.5)$ & $15(9.7)$ \\
$>60$ & 75 & $36(48)$ & $12(15)$ & $11(14.6)$ & $6(8)$ & $10(13.3)$ \\
\hline Totales & 266 & $136(51.1)$ & $25(9.3)$ & $46(17.2)$ & $32(12)$ & $27(10.1)$ \\
\hline
\end{tabular}

GRAFICA 5

CISTOSCOPIA / LDAD

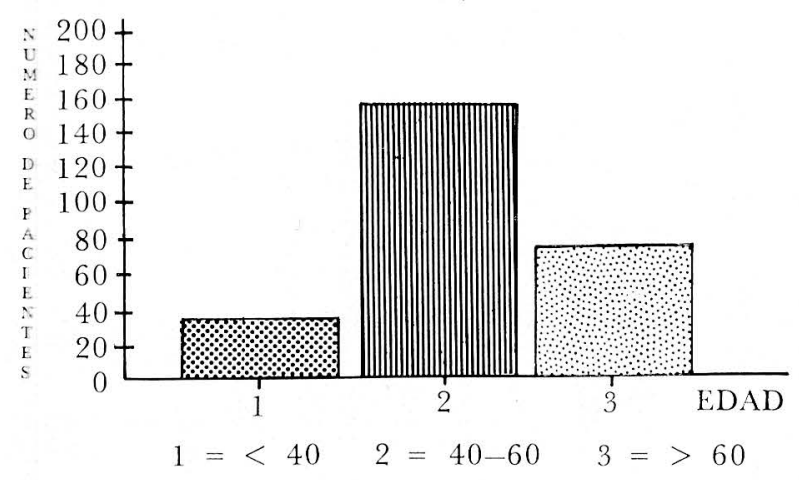

TABLA 8

UROGRAFIA / CISTOSCOPIA PRE-RADIOTERAPIA

\begin{tabular}{|ccccc|}
\hline Cistoscopia & Uro. N. & Uro. A2 & Uro. A3. & Uro. A4. \\
\hline N & 79 & 12 & 8 & 1 \\
A2 & 0 & 6 & 5 & 0 \\
A3 & 9 & 0 & 7 & 0 \\
A4 & 4 & 0 & 0 & 4 \\
\hline
\end{tabular}

TABLA 8A.

UROGRAFIA / CISTOSCOPIA PRE-RADIOTERAPIA

\begin{tabular}{|lcc|}
\hline Cistoscopia & Urografía normal & Urografía anormal \\
\hline Normal & 79 & 42 \\
Anormal & 54 & 56 \\
\hline
\end{tabular}

El resultado obtenido de la correlación entre biopsia vesical, síntomas y hematuria pre-radioterapia fue el siguiente:

TABLA 9

BIOPSIA VESICAL / SINTOMAS HEMATURIA. PRE-RADIO

\begin{tabular}{|lcccc|}
\hline $\begin{array}{l}\text { Biopsia } \\
\text { Vesical }\end{array}$ & $\begin{array}{c}\text { Síntomas } \\
\text { Si }\end{array}$ & $\begin{array}{c}\text { Síntomas } \\
\text { No }\end{array}$ & $\begin{array}{c}\text { Hematuria } \\
\text { Si }\end{array}$ & $\begin{array}{c}\text { Hematuria } \\
\text { No }\end{array}$ \\
\hline Positiva & 14 & 14 & 4 & 24 \\
Negativa & 2 & 23 & 0 & 25 \\
\hline
\end{tabular}


TABLA 10

SINTOMAS / HEMATURIA. PRE-POST-RADIOTERAPIA

\begin{tabular}{|ccccc|}
\hline \multirow{2}{*}{ Manifestación } & Síntomas & Síntomas Hematuria & \multicolumn{2}{c|}{ Hematuria } \\
\cline { 2 - 5 } & $\begin{array}{c}\text { pre-radio } \\
\text { No. }(\%)\end{array}$ & $\begin{array}{c}\text { post-radio } \\
\text { No. }(\%)\end{array}$ & $\begin{array}{c}\text { pre-radio } \\
\text { No. }(\%)\end{array}$ & $\begin{array}{c}\text { post-radio } \\
\text { No. }(\%)\end{array}$ \\
\hline $\mathrm{Si}$ & $39(13.5)$ & $101(35.1)$ & $8(2.7)$ & $4(1.3)$ \\
$\mathrm{No}$ & $248(86.4)$ & $186(64.8)$ & $279(97.2)$ & $283(98.6)$ \\
\hline
\end{tabular}

La correlación realizada entre sintomatología y hematuria pre y post-radioterapia nos reveló los siguientes datos: (Ver tabla 10).

La mortalidad observada después de terminado el tratamiento se discrimina de la siguiente manera:

Por causa urológica $\longrightarrow 19(6.62 \%)-$ IIIB $=16(84.2 \%)$

Por otras causas $\longrightarrow 9(3.1 \%) \quad$ IVB $=3(15.7 \%)$

Mortalidad total $\longrightarrow 28(.9 .7 \%)$

\section{GRAFICA 6}

MORTALIDAD / CAUSA

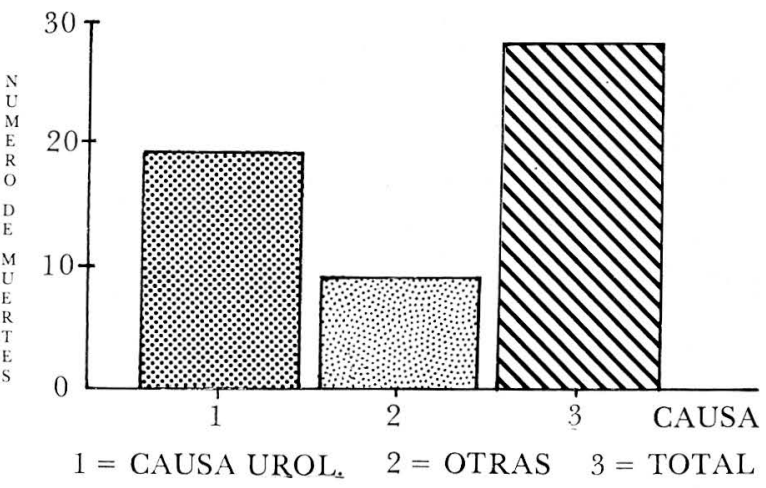

TABLA 11

MORTALIDAD / EDAD

\begin{tabular}{|crc|}
\hline Grupos de edad & No. Muertes & Porcentaje \\
\hline$<40$ & 1 & 5.2 \\
$40-60$ & 17 & 89.4 \\
$>60$ & 1 & 5.2 \\
\hline Totales & 19 & 100.0 \\
\hline
\end{tabular}

La correlación encontrada entre la mortalidad por causa urológica y los grupos de edad la vemos en la tabla 11.

GRAFICA 7

MORTALIDAD / EDAD

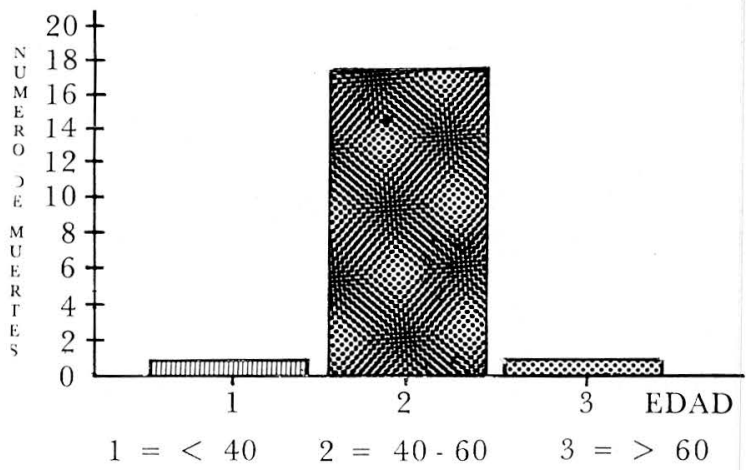

Así mismo, del cruce entre mortalidad y urografía obtuvimos:

TABLA 12

MORTALIDAD / UROGRAFIA

Hallazgos Urografía No. Muertes Porcentaje

$\begin{array}{lll}\mathrm{N} & 0 & 0\end{array}$

A1 115.2

$\begin{array}{lll}\mathrm{A} 2 & 0 & 0\end{array}$

$\begin{array}{lll}\text { A3 } & 6 & 31.5\end{array}$

$\begin{array}{lll}\text { A4 } & 12 & 63.1\end{array}$

Totales

19

100.0 
GRAFICA 8

MORT ALIDAD / UROGRAFIA

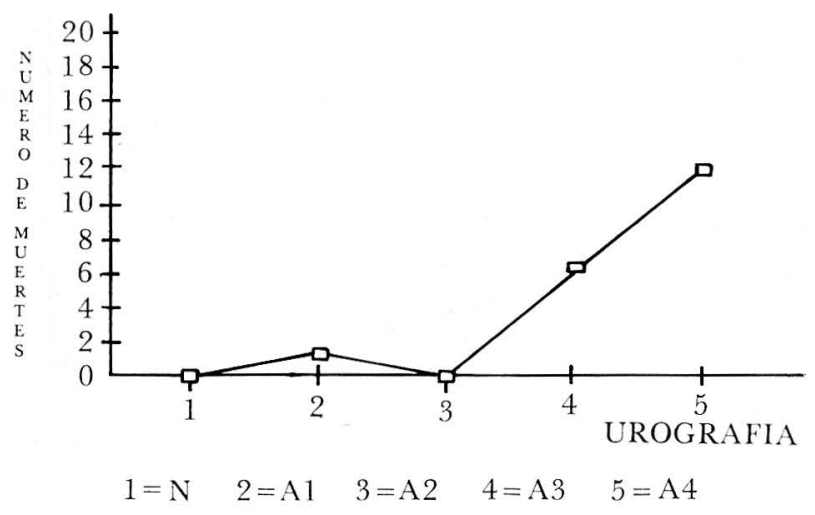

DISCUSION DE LOS RESULTADOS

E1 $13 \%$ de deserciones es un número muy alto a pesar de la entrevista social y de las múltiples llamadas a los pacientes por parte de la Trabajadora Social. Esto puede ser debido a la pésima información que tienen el médico y el paciente acerca de la curabilidad y la paliación de la enfermedad maligna. Por otro lado, el análisis de 287 pacientes tratados completamente en un sólo año con estados III y IV, con los mismos parámetros de estudio y con el mismo método terapéutico, da bases para obtener conclusiones válidas.

En la clasificación por edades y estados (tabla 1), observamos la clara preponderancia del estado IIIB y dentro de éste, del grupo intermedio de edades, grupo que por lo demás es el más frecuente en todos los estados. Cifra que es alarmante porque indica una clara deficiencia en el diagnóstico precoz.
Corroborando la importancia de la cistoscopia para definir, después del examen clínico, el estado del carcinoma del cervix, se aprecia que apenas la mitad de las cistoscopias fueron normales y de las anormales una cuarta parte tenían invasión a la mucosa vesical comprobada histológicamente (tabla 2).

De la comparación de las siguientes tres tablas que corresponden a nuestros resultados en relación con los obtenidos en las publicaciones del Journal of Urology por la Universidad de Arizona y los obtenidos por el Dr. Gaitán Yanguas en la Revista del Instituto Nacional de Cancerología, se enfatiza aún más la necesidad de realizar la cistoscopia en el carcinoma del cervix estados III y IV. Y así, pues, 22 pacientes pasaron de estado III a IV (Tabla 3 ).

TABLA 14

CARCINOMA DEL CERVIX (4)

\begin{tabular}{|lrrrrr|}
\hline Estado & Totales & Cis. N. & Biopsias & $\begin{array}{l}\text { Biopsias }+ \\
\text { Ca. Cervix } \\
\text { No. }(\%)\end{array}$ & $\begin{array}{l}\text { Biopsias }+ \\
\text { Ca. Cel. Trans. }\end{array}$ \\
\hline I & 37 & 35 & 2 & 0 & 0 \\
II & 41 & 39 & 2 & 0 & 0 \\
III & 25 & 12 & 13 & $3(12)$ & 1 \\
IV & 17 & 5 & 12 & $4(24)$ & 0 \\
\hline Totales & 120 & 91 & 29 & $7(6)$ & 1 \\
\hline
\end{tabular}

TABLA 15

CISTOSCOPIA / ESTADO CLINICO (5)

\begin{tabular}{|lcclllr|}
\hline Cistoscopia & Totales & In situ & I & II & III & IV \\
\hline Positiva & 30 & 0 & 0 & 0 & 22 & 8 \\
Negativa & 260 & 1 & 5 & 33 & 206 & 15 \\
\hline
\end{tabular}

TABLA 13

ESTADO / CISTOSCOPIA / BIOPSIA VESICAL (INC 1982)

\begin{tabular}{|lrllll|}
\hline Estado & Totales & $\begin{array}{l}\text { Cis. N. } \\
\text { No. }(\%)\end{array}$ & $\begin{array}{l}\text { Biopsias } \\
\text { No. }(\%)\end{array}$ & $\begin{array}{l}\text { Biopsias }+ \\
\text { Ca. Cervix } \\
\text { No. }(\%)\end{array}$ & $\begin{array}{l}\text { Biopsias }+ \\
\text { Ca. Cel. Trans. } \\
\text { No. }(\%)\end{array}$ \\
\hline III A & 3 & $2(0.7)$ & $1(0.3)$ & $1(0.3)$ & 0 \\
III B & 249 & $130(48.8)$ & $46(17.2)$ & $21(7.8)$ & 0 \\
IVA & 1 & $1(0.3)$ & 0 & 0 & 0 \\
IVB & 13 & $2(0.7)$ & $6(2.2)$ & $6(2.2)$ & 0 \\
\hline Totales & 266 & $135(50.7)$ & $53(19.9)$ & $28(10.5)$ & 0 \\
\hline
\end{tabular}


Con respecto a los resultados urográficos (tabla $4)$, vemos que hubo en los pacientes con estado IIIB aproximadamente un $60 \%$ de urografías normales y en cambio en el estado IVB solamente un $11 \%$. Dentro de las anormalidades la mayoría se encuentra en el grupo A3 (Exclusión renal unilateral o hidronefrosis bilateral), tanto en el estado III como en el estado IV, lo que indica un avanzado compromiso renal en estos pacientes. En cuanto a los grupos de edades hay clara preponderancia de los estados avanzados urográficos en los grupos menores de 40 años y mayores de 60 (tabla 5).

TABLA 16

OBSTRUCCION URETERAL PRE-RADIOTERAPIA POR CA DE CERVIX (6)

\begin{tabular}{|lcc|}
\hline Investigador & Pacientes & $\begin{array}{c}\text { Pacientes con } \\
\text { obstrucción } \\
\%\end{array}$ \\
\hline Aldridge and Mason (1950) & 333 & $115(34.5)$ \\
Burns et al. (1960) & 365 & $118(32.3)$ \\
Rhamy and Stander (1962) & 305 & $43(14.1)$ \\
Barber et al. (1963) & 503 & $105(20.9)$ \\
Kottmeier (1964) & 1.402 & $268(19.1)$ \\
Midboe et al. (1969) & 541 & $92(17.0)$ \\
Waggoner and Spratt (1969) & 945 & $215(22.8)$ \\
Bosch et al. (1973) & 990 & $143(14,4)$ \\
Vargas & 287 & $98(34.1)$ \\
\hline
\end{tabular}

De la lectura de esta tabla se deducen claramente varios hechos:

1. La Urografía simplificada es un instrumento adecuado de diagnóstico de las complicaciones urológicas producidas por el carcinoma de cervix ya que el porcentaje de positividad obtenido en nuestro estudio está en los índices más altos con respecto a las series extranjeras.

2. Que la circunstancia de alto índice de positividad es similar a las series más antiguas de los EE. UU. quizá porque dentro de los mismos estados avanzados (III y IV) el subgrupo con obstrucción urinaria tiene enfermedad más avanzada.

3. Que siendo igualmente eficaz es más barata que la urografía corriente usando sólo la mitad de las placas con el consiguiente ahorro de éstas, de equipo, de tiempo y sobre todo de radiación.

Al correlacionar los hallazgos cistoscópicos con el estado clínico (tabla 15), se observa un predominio de A2 y A3 en el estado III contra un predomi- nio de A4 en el estado IV. Y si tomamos las cistoscopias normales vemos que bajan del $66 \%$ en el estado IIIA al $52 \%$ en el estado IIIB y a $15 \%$ en el estado IVB, evidenciando una relación directa entre el grado de anormalidad cistoscópica y el estado clínico. En cuanto a la edad vemos una mayor incidencia de A4 cistoscópico en las pacientes mayores de 60 años y menores grados cistoscópicos en las menores de 40 (tabla 7).

Si entrecruzamos la urografía y la cistoscopia (tabla 8) vemos que cuando la urografía es normal hay mayor cantidad de cistoscopias normales que en el grupo de las urografías anormales. Cuando la cistoscopia es A4 la mitad tienen también Urografía A4. O sea, a mayor grado cistoscópico se aumenta notablemente no sólo el número de urografías anormales sino la severidad de los hallazgos de estas últimas y es cierto en forma inversa. A mayor grado de compromiso urográfico mayor porcentaje de anormalidad cistoscópica y dentro de ésta mayor porcentaje de los compromisos vesicales más severos.

Por otro lado hay estrecha relación entre los síntomas urinarios bajos y las biopsias positivas, más no así con la hematuria, posiblemente por la fácil confusión con el sangrado genital (tabla 9).

Los síntomas se agravan después del tratamiento radioterápico, aumentándose en un 300\% (tabla 10). En cambio la hematuria disminuyó porque la radioterapia es hemostática inicialmente, aunque como secuela puede dejar hematuria secundaria a cistitis post-irradiación.

Analizando la mortalidad antes del año post-tratamiento vemos que de las 28 pacientes que murieron en 19 la causa fue urológica, siendo pues el papel del urólogo el más importante. Dicho de otra forma el pronóstico de la paciente que recibió un tratamiento curativo está dado por el compromiso urinario y no por la extensión a los órganos genitales femeninos. Refiriéndonos a las edades, la mortalidad sigue el patrón de la incidencia (tabla 11) y al mirar la obstrucción ureteral observamos lógicamente que la mayoría de las urografías de las pacientes fallecidas por causa urológica eran A4 (tabla 12).

\section{CONCLUSIONES}

1. El estudio urológico mínimo después de diagnósticado un carcinoma de cervix comprende la urografía en todos los estados y la cistoscopia en los avanzados (III y IV). 
2. La urografía simplificada es suficiente para evaluar estas pacientes.

3. Cuando hay anormalidad es conveniente la utilización de estudios isotópicos.

4. El pronóstico de la sobrevida está dado básicamente por el compromiso urinario.

5. Los síntomas urinarios bajos sugieren compromiso tumoral vesical. Contrariamente la hematuria no se relaciona con la invasión tumoral.

6. Las severas anormalidades urográficas se asocian con severas anormalidades cistoscópïcas.

7. Con respecto a la edad en la cual se presenta más frecuentemente en nuestro medio el carcinoma de cervix avanzado, hay más anormalidades urográficas en los grupos más jóvenes y más viejos que además tienen la menor rata de incidencia de la enfermedad.
8. Después de diagnosticado el carcinoma de cervix los estudios urológicos son los más importantes para determinar el estadio real de avance de la enfermedad y para dar el pronóstico y aislar así el grupo de pacientes con más alto riesgo y de esta manera, poder darles un tratamien to adecuado.

\section{RESUMEN}

En el Instituto Nacional de Cancerología en el año de 1982 se atendieron 8.586 pacientes nuevos, de los cuales se comprobó cáncer en 3.480 y carcinoma de cervix en 895 . Estado clínico III 350 pacientes y estado IV 35 pacientes. Se estudiaron con urografía y cistoscopia y muchas de ellas con estudios gamagráficos y biopsias vesicales cuando estaba indicado antes de tratamiento. Las pacientes se siguieron por un año que se consideró era el período temprano de seguimiento. Se presentan, discuten y analizan las complicaciones presentadas en estas pacientes.

\section{BIBLIOGRAFIA}

1. Registro Nacional del Cáncer INC.

2. BOSCH, A., FriAs, Z., and de VALDA, G.C.: Pronostic Significance of ureteral obstruction in $\mathrm{Ca}$. of the Cervix Uteri. Acta Radiol. 12: 47, 1973.

3. BUCHSBAUM and SCHMIDT.: Gynecologic and Obstetric Urology. Philadelphia, W.B. Saunders Company, 1982.

4. ROMERO, R.E., HICKS, T.H., GALINDO, G.H. and DRACH, G.W.: Evaluation of importance of cystoscopy in staging gynecologic carcinomas. The J. of Urol., $121: 64,1979$.

5. GAITAN YANGUAS, M. y BUENO-PONCE, C.: La endoscopia rectal y vesical en la clasificación del cán- cer de cuello uterino. Se justifica su uso rutinario. Rev. Inst. Nal. Cancerol. Col. 1: 1, 59, 1980.

6. ALDRIDGE, C.W., and MASON, J.T.: Ureteral obstruction in carcinoma of the cervix. Am. J. Obstet. Gynecol. 60: 1272, 1950.

7. BARBER, H.R.K., ROBERTS, S., and BRUNSCHWIG, A.: Prognostic significance of preoperative nonvisualizing Kidney in patients receiving pelvic exenteration. Cancer 16: 1614, 1963.

8. BURNS, B.C., EVERETT, H.S., and BRACK, C.B.: Value of urologic study in the management of carcinoma of the cervix. Am. J. Obstet. Gynecol. 80: 997, 1960. 